\title{
A NOTE ON INTEGRAL MODIFICATION OF THE MEYER-KÖNIG AND ZELLER OPERATORS
}

\author{
VIJAY GUPTA and NIRAJ KUMAR
}

Received 6 August 2002

Guo (1988) introduced the integral modification of Meyer-König and Zeller operators $\hat{M}_{n}$ and studied the rate of convergence for functions of bounded variation. Gupta (1995) gave the sharp estimate for the operators $\hat{M}_{n}$. Zeng (1998) gave the exact bound and claimed to improve the results of Guo and Gupta, but there is a major mistake in the paper of Zeng. In the present note, we give the correct estimate for the rate of convergence on bounded variation functions.

2000 Mathematics Subject Classification: 41A30, 41A36.

1. Introduction. For a function defined on $[0,1]$, the Meyer-König and Zeller operators $P_{n}, n \in \mathbb{N}$, [8] are defined by

$$
P_{n}(f, x)=\sum_{k=0}^{\infty} p_{n, k}(x) f\left(\frac{k}{n+k}\right), \quad x \in[0,1],
$$

where

$$
p_{n, k}(x)=\left(\begin{array}{c}
n+k-1 \\
k
\end{array}\right) x^{k}(1-x)^{n}
$$

The rates of convergence of some integral modifications of operators (1.1) were discussed in [2, 4, 5, 6, 7, 10]. Guo [1] introduced Meyer-König and Zeller operators as

$$
\hat{M}_{n}(f, x)=\sum_{k=1}^{\infty} p_{n, k+1}(x) \frac{(n+k-1)(n+k-3)}{(n-2)} \int_{0}^{1} p_{n-2, k-1}(t) f(t) d t,
$$

where $p_{n, k}(x)$ is defined in (1.2).

Guo [1] obtained the following theorem.

THEOREM 1.1. Let $f$ be a function of bounded variation on $[0,1], x \in(0,1)$. Then, for all $n$ sufficiently large,

$$
\begin{aligned}
\left|\hat{M}_{n}(f, x)-\frac{1}{2}\left\{f\left(x^{+}\right)+f\left(x^{-}\right)\right\}\right| \leq & \frac{7}{n x} \sum_{k=1}^{n} V_{x-x / \sqrt{k}}^{x+(1-x) / \sqrt{k}}\left(g_{x}\right) \\
& +\frac{50}{\sqrt{n} \cdot x^{3 / 2}}\left|f\left(x^{+}\right)-f\left(x^{-}\right)\right|,
\end{aligned}
$$


where

$$
g_{x}(t)= \begin{cases}f(t)-f\left(x^{-}\right), & 0 \leq t<x \\ 0, & t=x \\ f(t)-f\left(x^{+}\right), & x<t \leq 1\end{cases}
$$

and $V_{a}^{b}\left(g_{x}\right)$ is the total variation of $g_{x}$ on $[a, b]$.

Gupta [3] gave a sharp estimate as in the following theorem.

THEOREM 1.2. Let $f$ be a function of bounded variation on $[0,1], x \in(0,1)$. Then, for all $n$ sufficiently large,

$$
\begin{aligned}
\left|\hat{M}_{n}(f, x)-\frac{1}{2}\left\{f\left(x^{+}\right)+f\left(x^{-}\right)\right\}\right| \leq & \frac{7}{n x} \sum_{k=1}^{n} V_{x-x / \sqrt{k}}^{x+(1-x) / \sqrt{k}}\left(g_{x}\right) \\
& +\frac{114}{15 \sqrt{n} \cdot x^{3 / 2}}\left|f\left(x^{+}\right)-f\left(x^{-}\right)\right| .
\end{aligned}
$$

Zeng [9] gave the exact bound for Meyer-König and Zeller basis functions and claimed to obtain the sharp estimate over the results of Guo [1] and Gupta [3]. Although the bound obtained in [9] is optimum, the main estimate given by Zeng [9] for the operators $\hat{M}_{n}(f, x)$ is not correct. Zeng obtained the following theorem.

THEOREM 1.3. Let $f$ be a function of bounded variation on $[0,1]$. Then, for every $x \in(0,1)$ and $n$ sufficiently large,

$$
\begin{aligned}
\left|\hat{M}_{n}(f, x)-\frac{1}{2}\left\{f\left(x^{+}\right)+f\left(x^{-}\right)\right\}\right| \leq & \frac{7}{n x} \sum_{k=1}^{n} V_{x-x / \sqrt{k}}^{x+(1-x) / \sqrt{k}}\left(g_{x}\right) \\
& +\frac{3}{\sqrt{8 e} \cdot x^{3 / 2}}\left|f\left(x^{+}\right)-f\left(x^{-}\right)\right| .
\end{aligned}
$$

We may remark here that Theorem 1.3 obtained by Zeng [9] has a major mistake because the right-hand side does not converge to zero for sufficiently large $n$. Also, the remark given before [9, Theorem 4.3] is contradictory to the main theorem [9, Theorem 4.3]. This motivated us to give the correct estimate for these operators and in this note we give an improved estimate for the rate of convergence on functions of bounded variation for the operators (1.3).

2. Auxiliary results. In this section, we give certain results, which are necessary to prove the main result.

LEMмA 2.1 [7]. Let $X_{1}, X_{2}, X_{3}, \ldots, X_{n}$ be $n$ independent and identically distributed random variables with zero mean and a finite absolute third moment. If $\rho_{2}=E\left(X_{1}^{2}\right)>0$, then

$$
\sup _{x \in R}\left|F_{n}(x)-\Phi(x)\right| \leq(0.409) \ell_{3, n},
$$


where $F_{n}$ is the distribution function and $\ell_{3, n}$ is the Liapounov ratio given by

$$
\ell_{3, n}=\left(\frac{\rho_{3}}{\rho_{2}^{3 / 2}}\right), \quad \rho_{3}=E\left(\left|X_{1}\right|^{3}\right) .
$$

LEMMA 2.2 [7]. If $\left\{\xi_{i}\right\}, i=1,2,3, \ldots$, are independent random variables with the same geometric distribution

$$
P\left(\xi_{i}\right)=x^{k}(1-x), \quad x \in(0,1), i=1,2,3, \ldots,
$$

then

$$
E\left(\xi_{i}\right)=\frac{x}{1-x}, \quad \rho_{2}=E\left(\xi_{i}-E\left(\xi_{i}\right)\right)^{2}=\frac{x}{(1-x)^{2}}
$$

and $\eta_{n}=\sum_{i=1}^{n} \xi_{i}$ is a random variable with distribution

$$
P\left(\eta_{n}=k\right)=\left(\begin{array}{c}
n+k-1 \\
k
\end{array}\right) x^{k}(1-x)^{n}
$$

LEMMA 2.3 [9]. For all $k, n \in \mathbb{N}$ and $x \in(0,1]$,

$$
p_{n, k}(x)<\frac{1}{\sqrt{2 e} \sqrt{n} x^{3 / 2}},
$$

where the constant $1 / \sqrt{2 e}$ is the best possible.

LEMMA 2.4. For $k \geq 1, x \in(0,1)$, and $n>2$,

$$
\left|\sum_{j=2}^{k+1} p_{n, j}(x)-\sum_{j=1}^{k-1} p_{n-1, j}(x)\right| \leq \frac{3}{\sqrt{n} x^{3 / 2}}
$$

Proof. By (2.5), we have

$$
\begin{aligned}
p_{n, k}(x) & =P\left(\eta_{n}=k\right)=P\left(k-1<\eta_{n} \leq k\right) \\
& =P\left(\frac{k-1-n x /(1-x)}{\sqrt{n x} /(1-x)}<\frac{\eta_{n}-n x /(1-x)}{\sqrt{n x} /(1-x)} \leq \frac{k-n x /(1-x)}{\sqrt{n x} /(1-x)}\right) .
\end{aligned}
$$

Using Lemma 2.1, we obtain

$$
\left|P\left(\eta_{n}=k\right)-\frac{1}{\sqrt{2 \pi}} \int_{(k-1-n x /(1-x)) /(\sqrt{n x} /(1-x))}^{(k-n x /(1-x)) /(\sqrt{n x} /(1-x))} e^{-t^{2} / 2} d t\right|<\frac{2(0.409) \rho_{3}}{\sqrt{n} \rho_{2}^{3 / 2}} .
$$


Next, we estimate $\rho_{3}$. For $T_{r}(x)=\sum_{k=0}^{\infty} k^{r} x^{k}(1-x)$, we have by an easy computation

$$
\begin{aligned}
& T_{0}(x)=1, \quad T_{1}(x)=\frac{x}{1-x}, \quad T_{2}(x)=\frac{x(1+x)}{(1-x)^{2}} \\
& T_{3}(x)=\frac{x^{3}+4 x^{2}+x}{(1-x)^{3}}, \quad T_{4}(x)=\frac{x^{4}+11 x^{3}+11 x^{2}+x}{(1-x)^{4}} .
\end{aligned}
$$

Also, if $M_{r}(x)=\sum_{k=0}^{\infty}(k-x /(1-x))^{r} x^{k}(1-x)$ stands for the central moment of order $r$ about the mean $x /(1-x)$, it is easily checked by using the above that

$$
\begin{aligned}
& M_{2}(x)=\sum_{j=0}^{2}\left(\begin{array}{l}
2 \\
j
\end{array}\right)(-1)^{j} T_{2-j}(x)\left(T_{1}(x)\right)^{j}=\frac{x}{(1-x)^{2}}, \\
& M_{4}(x)=\sum_{j=0}^{4}\left(\begin{array}{l}
4 \\
j
\end{array}\right)(-1)^{j} T_{4-j}(x)\left(T_{1}(x)\right)^{j}=\frac{x^{3}+7 x^{2}+x}{(1-x)^{4}} .
\end{aligned}
$$

Thus, using the inequality $\rho_{3} \leq\left(M_{2}(x) M_{4}(x)\right)^{1 / 2}$, we have

$$
\rho_{3} \leq\left(\frac{x}{(1-x)^{2}} \cdot \frac{x^{3}+7 x^{2}+x}{(1-x)^{4}}\right)^{1 / 2} \leq \frac{3}{(1-x)^{3}}, \quad x \in(0,1) .
$$

So, the right-hand side of (2.9) is less than $2.454 / \sqrt{n} x^{3 / 2}$, that is, less than $5 / 2 \sqrt{n} x^{3 / 2}$.

Also, since

$$
\sum_{j=0}^{k+1} p_{n, j}(x)=P\left(\eta_{n} \leq k+1\right), \quad \sum_{j=0}^{k-1} p_{n-1, j}(x)=P\left(\eta_{n-1} \leq k-1\right),
$$

thus

$$
\begin{array}{|}
\left|\sum_{j=0}^{k+1} p_{n, j}(x)-\frac{1}{\sqrt{2 \pi}} \int_{-\infty}^{(k+1-n x /(1-x)) /(\sqrt{n x} /(1-x))} e^{-t^{2} / 2} d t\right|<\frac{5}{4 \sqrt{n} x^{3 / 2}}, \\
\left|\sum_{j=0}^{k-1} p_{n-1, j}(x)-\frac{1}{\sqrt{2 \pi}} \int_{-\infty}^{(k-1-(n-1) x /(1-x)) /(\sqrt{(n-1) x} /(1-x))} e^{-t^{2} / 2} d t\right| \\
<\frac{5}{4 \sqrt{(n-1)} x^{3 / 2}} .
\end{array}
$$


On the other hand,

$$
p_{n, 0}(x)+p_{n, 1}(x)=o\left(n^{-1}\right) .
$$

Hence, for $n$ sufficiently large, we have

$$
\begin{aligned}
\left|\sum_{j=2}^{k+1} p_{n, j}(x)-\sum_{j=0}^{k-1} p_{n-1, j}(x)\right| & <\frac{10}{4 \sqrt{n} x^{3 / 2}}+\frac{1}{\sqrt{2 \pi}} \int_{I_{n, k}} e^{-t^{2} / 2} d t \\
& <\frac{5}{2 \sqrt{n} x^{3 / 2}}+\frac{1-x}{\sqrt{2 \pi n x}} \\
& <\frac{5}{2 \sqrt{n} x^{3 / 2}}+\frac{1}{2 \sqrt{n} x^{3 / 2}} \\
& =\frac{3}{\sqrt{n} x^{3 / 2}},
\end{aligned}
$$

where

$$
I_{n, k}=\left[\frac{k-1-n x /(1-x)}{\sqrt{n x} /(1-x)}, \frac{k-n x /(1-x)}{\sqrt{n x} /(1-x)}\right] .
$$

This completes the proof of the lemma.

LEMMA 2.5 [1]. For $k \geq 1, x \in(0,1)$, and $n>2$,

$$
\sum_{j=0}^{k-1} p_{n-1, j}(x)=\frac{(n+k-2)(n+k-3)}{(n-2)} \int_{x}^{1} p_{n-2, k-1}(t) d t .
$$

3. Main result. In this section, we prove the following main theorem.

THEOREM 3.1. Let $f$ be a function of bounded variation on $[0,1]$. Then, for every $x \in(0,1)$ and $n$ sufficiently large,

$$
\begin{aligned}
\left|\hat{M}_{n}(f, x)-\frac{1}{2}\left\{f\left(x^{+}\right)+f\left(x^{-}\right)\right\}\right| \leq & \frac{7}{n x} \sum_{k=1}^{n} V_{x-x / \sqrt{k}}^{x+(1-x) / \sqrt{k}}\left(g_{x}\right) \\
& +\left(3+\frac{1}{\sqrt{8 e}}\right) \frac{1}{\sqrt{n} x^{3 / 2}}\left|f\left(x^{+}\right)-f\left(x^{-}\right)\right|,
\end{aligned}
$$

where $V_{a}^{b}\left(g_{x}\right)$ is the total variation of $g_{x}$ on $[a, b]$.

Proof. First,

$$
\begin{aligned}
& \left|\hat{M}_{n}(f, x)-\frac{1}{2}\left\{f\left(x^{+}\right)+f\left(x^{-}\right)\right\}\right| \\
& \quad \leq\left|\hat{M}_{n}\left(g_{x}, x\right)\right|+\frac{1}{2}\left|f\left(x^{+}\right)-f\left(x^{-}\right)\right| \cdot\left|\hat{M}_{n}(\operatorname{sign}(t-x), x)\right| .
\end{aligned}
$$


Now, with the kernel

$$
K_{n}(x, t)=\sum_{k=1}^{\infty} \frac{(n+k-2)(n+k-3)}{(n-2)} p_{n, k+1}(x) p_{n-2, k-1}(t),
$$

we have

$$
\begin{aligned}
\hat{M}_{n}(\operatorname{sign}(t-x), x) & =\int_{0}^{1} K_{n}(x, t) \operatorname{sign}(t-x) d t \\
& =\int_{x}^{1} K_{n}(x, t) d t-\int_{0}^{x} K_{n}(x, t) d t \\
& =A_{n}(x)-B_{n}(x) .
\end{aligned}
$$

Using Lemma 2.5, we have

$$
\begin{aligned}
A_{n}(x) & =\int_{x}^{1} K_{n}(x, t) d t \\
& =\sum_{k=1}^{\infty} p_{n, k+1}(x) \frac{(n+k-2)(n+k-3)}{(n-2)} \int_{x}^{1} p_{n-2, k-1}(t) d t \\
& =\sum_{k=1}^{\infty}\left(p_{n, k+1}(x) \sum_{j=0}^{k-1} p_{n-1, j}(x)\right) .
\end{aligned}
$$

Using Lemma 2.4, we get

$$
\left|A_{n}(x)-\sum_{k=1}^{\infty}\left(p_{n, k+1}(x) \sum_{j=2}^{k+1} p_{n, j}(x)\right)\right| \leq \frac{3}{\sqrt{n} x^{3 / 2}} .
$$

Next, by Lemma 2.3, we get

$$
\left|\sum_{k=1}^{\infty}\left(p_{n, k+1}(x) \sum_{j=2}^{k+1} p_{n, j}(x)\right)-\frac{1}{2}\right| \leq \frac{1}{2 \sqrt{2 e} \sqrt{n} x^{3 / 2}} .
$$

Hence,

$$
\begin{aligned}
\left|\hat{M}_{n}(\operatorname{sign}(t-x), x)\right| & =\left|A_{n}(x)-B_{n}(x)\right| \\
& =\left|2 A_{n}(x)-1+o\left(n^{-1}\right)\right| \\
& \leq 2\left(3+\frac{1}{\sqrt{8 e}}\right) \frac{1}{\sqrt{n} x^{3 / 2}}+o\left(n^{-1}\right) .
\end{aligned}
$$

Substituting the value of $\left|\hat{M}_{n}(\operatorname{sign}(t-x), x)\right|$ and proceeding along the lines of [1] for the value of $\left|\hat{M}_{n}\left(g_{x}, x\right)\right|$, the theorem follows.

This completes the proof of theorem.

REMARK 3.2. We remark here that in order to prove the main theorem, the inequality $\rho_{3} \leq 16 /(1-x)^{3}$ is used in Theorem 1.1 and the value $\rho_{3} \leq 3 \sqrt{3} /(1-x)^{3}$ 
is used in Theorem 1.2. Zeng [9] used this value and the exact bound and gave the misprinted theorem (Theorem 1.3). Although Zeng obtained the exact bound for Meyer-König and Zeller basis functions, he has not used it correctly to obtain his main result, that is, Theorem 1.3.

4. Bezier variant of the operators $\hat{M}_{n}$. In this section, we propose the Bezier variant of the integrated Meyer-König and Zeller operators as

$$
\hat{M}_{n, \alpha}(f, x)=\sum_{k=1}^{\infty} Q_{n, k+1}^{(\alpha)}(x) \frac{(n+k-1)(n+k-3)}{(n-2)} \int_{0}^{1} p_{n-2, k-1}(t) f(t) d t,
$$

where $\alpha \geq 1, Q_{n, k}^{(\alpha)}(x)=\left(\sum_{j=k}^{\infty} p_{n, j}(x)\right)^{\alpha}-\left(\sum_{j=k+1}^{\infty} p_{n, j}(x)\right)^{\alpha}, \hat{M}_{n, \alpha}(1, x)=1$. It is easily verified that the operators (4.1) are linear positive operators. In particular, for $\alpha=1$, operators (4.1) reduce to the operators (1.3).

\section{REFERENCES}

[1] S. S. Guo, Degree of approximation to functions of bounded variation by certain operators, Approx. Theory Appl. 4 (1988), no. 2, 9-18.

[2] _ On the rate of convergence of the integrated Meyer-König and Zeller operators for functions of bounded variation, J. Approx. Theory 56 (1989), no. 3, 245-255.

[3] V. Gupta, A sharp estimate on the degree of approximation to functions of bounded variation by certain operators, Approx. Theory Appl. (N.S.) 11 (1995), no. 3, 106-107.

[4] _ A note on Meyer-König and Zeller operators for functions of bounded variation, Approx. Theory Appl. (N.S.) 18 (2002), no. 3, 99-102.

[5] _ On a new type of Meyer-Konig and Zeller operators, JIPAM. J. Inequal. Pure Appl. Math. 3 (2002), no. 4, Article 57, 1-10.

[6] V. Gupta and U. Abel, Rate of convergence by a new type of Meyer König and Zeller operators, to appear in Fasc. Math.

[7] E. R. Love, G. Prasad, and A. Sahai, An improved estimate of the rate of convergence of the integrated Meyer-König and Zeller operators for functions of bounded variation, J. Math. Anal. Appl. 187 (1994), no. 1, 1-16.

[8] W. Meyer-König and K. Zeller, Bernsteinsche Potenzreihen, Studia Math. 19 (1960), 89-94 (German).

[9] X. Zeng, Bounds for Bernstein basis functions and Meyer-König and Zeller basis functions, J. Math. Anal. Appl. 219 (1998), no. 2, 364-376.

[10] _ Rates of approximation of bounded variation functions by two generalized Meyer-König and Zeller type operators, Comput. Math. Appl. 39 (2000), no. 9-10, 1-13.

Vijay Gupta: School of Applied Sciences, Netaji Subhas Institute of Technology, Azad Hind Fauj Marg, Sector 3 Dwarka, Pappankalan, New Delhi 110 045, India

E-mail address: vijay@nsit.ac.in

Niraj Kumar: School of Applied Sciences, Netaji Subhas Institute of Technology, Azad Hind Fauj Marg, Sector 3 Dwarka, Pappankalan, New Delhi 110 045, India

E-mail address: neeraj@nsit.ac. in 


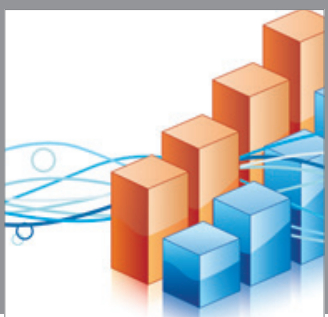

Advances in

Operations Research

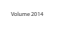

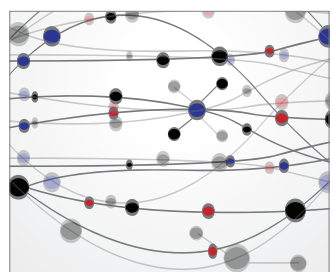

\section{The Scientific} World Journal
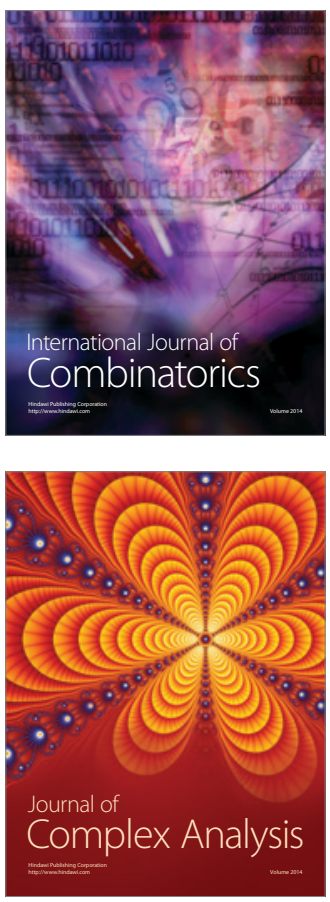

International Journal of

Mathematics and

Mathematical

Sciences
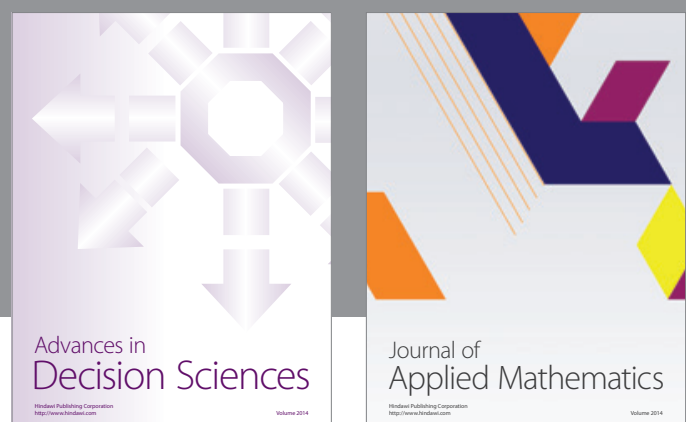

Journal of

Applied Mathematics
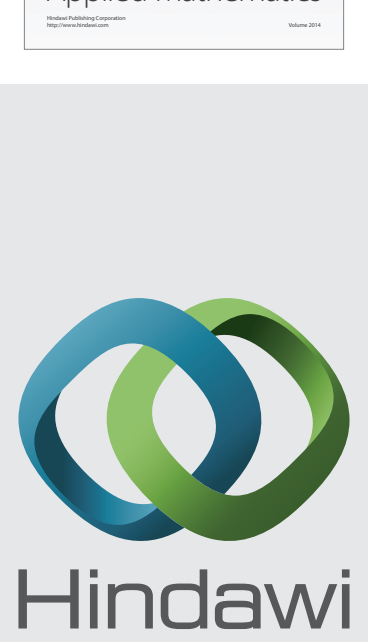

Submit your manuscripts at http://www.hindawi.com
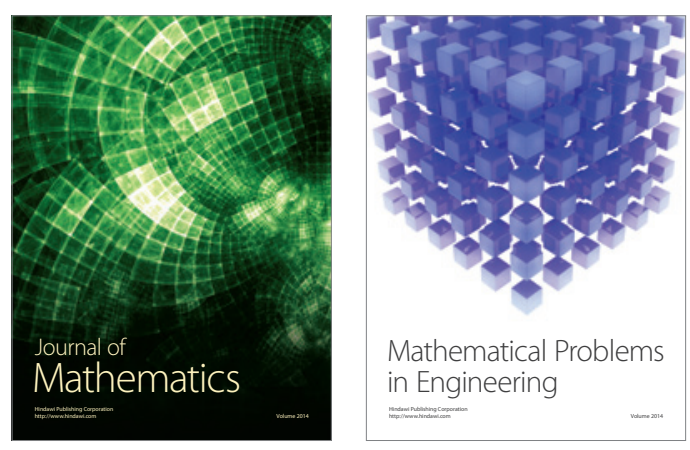

Mathematical Problems in Engineering
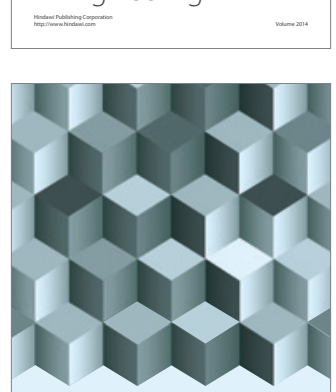

Journal of

Function Spaces
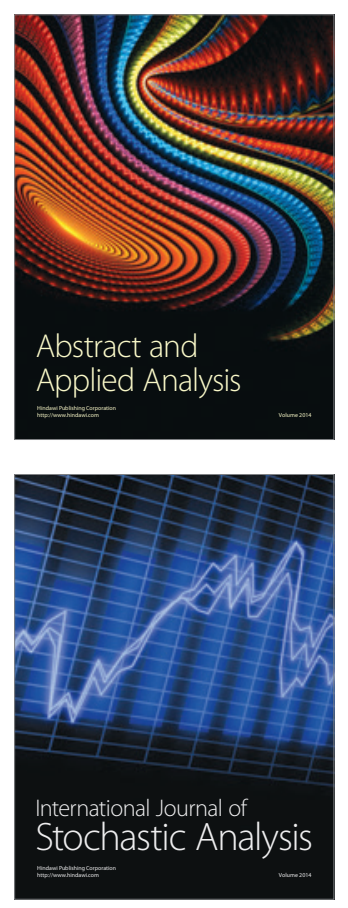

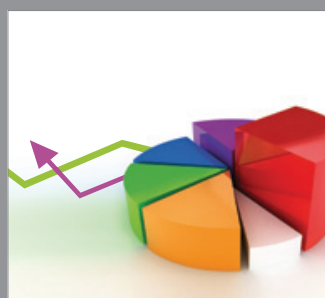

ournal of

Probability and Statistics

Promensencen
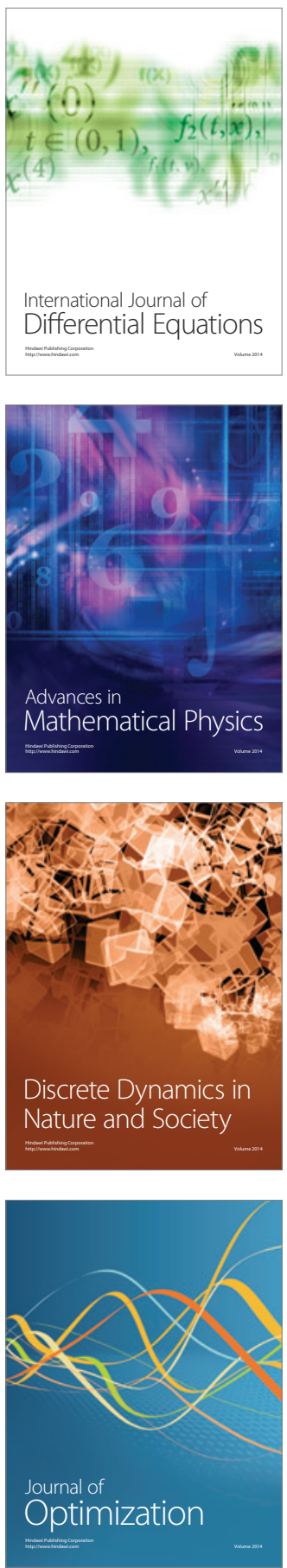